\title{
Evolução da estratégia Saúde da Família e sua influência nos indicadores da Atenção Básica em Saúde no Estado de Mato Grosso
}

Evolution of the Family Health Strategy and influence on the Basic Health Care indicators in the State of Mato Grosso

Evolución de la Estrategia de Salud de la Familia y su influencia en los indicadores de Atención Primaria de Salud en el Estado de Mato Grosso

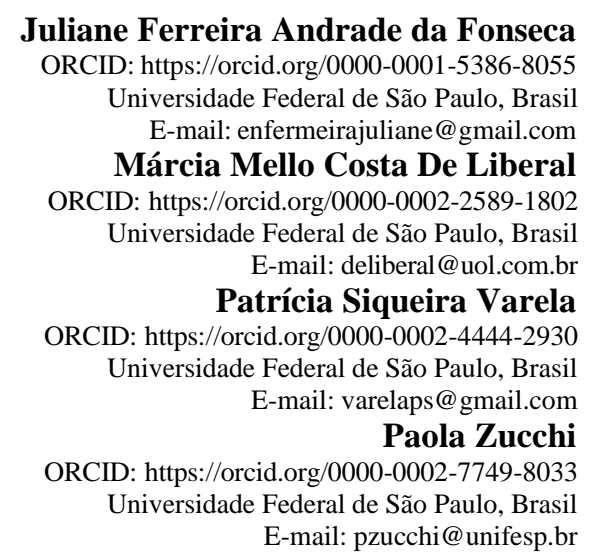

\section{Resumo}

Este estudo teve como objetivo correlacionar os indicadores de Atenção Básica em Saúde nos seus componentes de estrutura com os de processo e também com os resultados nos municípios de Mato Grosso, de 2008 a 2015. Trata-se de uma pesquisa avaliativa, quantitativa, retrospectiva com uso de dados secundários oriundos dos sistemas de informações. Foi construída uma matriz composta por componentes da estrutura (cobertura populacional potencial), do processo (consulta médica, visita domiciliar de médico e enfermeiro, encaminhamento à especialista e solicitação de exames de patologia clínica) e do resultado (taxa de internação por causas sensíveis a atenção básica, pela proporção de nascidos vivos de mães com sete ou mais consultas de pré-natal e pelo coeficiente de mortalidade infantil) e foi feita a análise descritiva e coeficiente de Correlação de Spearman (rho). O estudo apontou que a cobertura populacional manteve alta, acima de $83 \%$ e os indicadores de processo sugerem uma melhora da estratégia saúde da família, com redução de $63,13 \%$ na média de encaminhamento à especialista e de 49,71\% na solicitação de exames de patologia clínica. Porém, houve uma redução de $7,13 \%$ na média de visita domiciliar no período estudado. Existe correlação entre o componente de estrutura e processo e entre estrutura e resultado. Verificou-se que, com a evolução da Estratégia Saúde da Família, ocorreram alterações em alguns indicadores da atenção básica, entretanto, não é possível afirmar que houve mudança no modelo assistencial.

Palavras-chave: Estratégia saúde da família; Indicadores básicos de saúde; Avaliação em saúde; Gestão em saúde.

\begin{abstract}
This research aimed to correlate the indicators of Primary Health Care in their structure components with those of the process and with the results in the municipalities of Mato Grosso, Brazil, from 2008 to 2015. This is an evaluative, quantitative, retrospective research with use of secondary data from information systems. A matrix is composed by components of the structure (potential population coverage) the process (medical consultation, home visit of doctor and nurse, referral to the specialist and request of clinical pathology exams) and the result (hospitalization rate due to sensitive causes). It was construct when primary care, the proportion of live births of mothers with seven or more prenatal consultations and the infant mortality coefficient) and the descriptive analysis and Spearman correlation coefficient (rho) were performed. The study found that population coverage remained high above $83 \%$ and the process indicators suggest an improvement in family health strategy, with a reduction of $63.13 \%$ in the average referral to a specialist and $49.71 \%$ in the request for clinical pathology exams. However, there was a $7.13 \%$ reduction in the average home visit during the study period. There is a correlation between the structure and process component and between structure and result. It has found that with the evolution of the Family Health Strategy (FHS), there were changes in
\end{abstract}


some indicators of primary care, but it is not possible to state that there was a change in the care model. Keywords: Family health strategy; Basic health indicators; Health evaluation; Health management.

\section{Resumen}

Este estudio tuvo como objetivo correlacionar los indicadores de Atención Primaria de Salud en sus componentes de estructura con el proceso y también con los resultados de los municipios de Mato Grosso, de 2008 a 2015. Se trata de una investigación evaluativa, cuantitativa, retrospectiva con uso de datos secundarios de información. sistemas. Se construyó una matriz compuesta por componentes de la estructura (cobertura de población potencial), el proceso (cita médica, visita domiciliaria de médico y enfermera, derivación a especialista y solicitud de pruebas de patología clínica) y el resultado (tasa de ingreso por determinadas causas). a atención primaria, por la proporción de nacidos vivos a madres con siete o más visitas prenatales y por el coeficiente de mortalidad infantil) y se realizó un análisis descriptivo y el coeficiente de correlación de Spearman (rho). El estudio señaló que la cobertura poblacional se mantuvo alta, por encima del $83 \%$ y los indicadores del proceso evolutivo una mejora en la estrategia de salud de la familia, con una reducción del 63,13\% en la derivación promedio a un especialista y del 49,71\% en la solicitud de exámenes de patología clínica. Sin embargo, hubo una reducción del 7,13\% en el promedio de visitas domiciliarias durante el período de estudio. Existe una correlación entre el componente de estructura y el proceso y entre la estructura y el resultado. Se encontró que, con la evolución de la Estrategia Salud de la Familia, hubo cambios en algunos indicadores de la atención primaria, sin embargo, no es posible decir que hubo un cambio en el modelo de atención.

Palabras clave: Estrategia de salud de la familia; Indicadores básicos de salud; Valoración de salud; Manejo de la salud.

\section{Introdução}

A Estratégia Saúde da Família (ESF) foi implementada, no Brasil, pelo Ministério da Saúde (MS) no início da década de 1990, a partir de experiências exitosas internacionais, referentes aos critérios estabelecidos na Conferência de Alma Ata para a Atenção Primária em Saúde, baseada nos princípios do acesso universal, o cuidado continuado, integral e coordenado nas comunidades através de atividades programadas, grupos terapêuticos, visitas, internações domiciliares, dentre outras, indo além do atendimento médico individual (Soratto et al., 2015).

A ESF configura-se como uma proposta de reorganização da Atenção Básica em Saúde (ABS) e consolidação dos princípios do Sistema Único de Saúde (SUS) impulsionando mudanças na forma de organização dos serviços, das práticas assistenciais, centrada na família, em seu contexto físico, social e em expandir a rede de serviços assistenciais (Soratto et al., 2015). A implementação da ESF, na perspectiva da reorganização do modelo assistencial, teve características inovadoras, o que a diferencia do modelo de saúde tradicional. A organização do processo de trabalho, a partir de uma equipe multiprofissional, assume a responsabilidade por, no máximo, quatro mil pessoas, sendo a média recomendada de 3.000 pessoas, em espaço geográfico restrito. Essa equipe tem ampliado o vínculo e a corresponsabilidade entre as famílias e comunidade por meio de práticas de cuidado humanizado e integral (Brasil, 2012; Motta \& Siqueira-Batista, 2015).

A ESF atua na atenção básica de maneira contínua com foco na família e na comunidade. No desenvolvimento de suas atividades, a equipe de saúde da família elabora relatórios com informações referentes à situação sanitária e processo de trabalho. Esses dados são registrados no Sistema de Informação da Atenção Básica (SIAB), tornando-se indicadores de saúde que subsidiarão o planejamento das ações prioritárias às necessidades de saúde (Senna \& Andrade, 2015). Essa iniciativa teve uma rápida expansão em todo o território brasileiro, mas sua implementação não ocorreu de forma homogênea nos diversos municípios brasileiros, o que tem limitado seu avanço para consolidação e reorganização efetiva do SUS, uma vez que sua execução depende do apoio do governo federal, estadual, da política municipal, da adesão e do desempenho dos profissionais das equipes de saúde da família (Motta \& Siqueira-Batista, 2015). Com a intenção de acompanhar e monitorar as ações de saúde realizadas na ABS cujos resultados refletem na saúde da população, a partir de 2006, por meio do Pacto pela Saúde, houve a pactuação de indicadores da atenção básica que estão relacionados às áreas prioritárias do Pacto pela Vida e do Pacto de Gestão. Esses indicadores permitem visualizar modificações no contexto socioeconômico, no desempenho dos serviços de saúde, condições de vida e saúde da população (Sala \& Mendes, 2011). 
No levantamento realizado sobre a avaliação da implantação da ESF, percebeu-se que a mesma sofre avaliações díspares quanto às suas características relacionadas às dimensões Político-Institucional, Organizativa e Técnico-Assistencial. A dimensão político-institucional envolve elementos da expansão, equidade, financiamento, formação e gestão de pessoas. A organizativa é composta por elementos do acesso, integralidade e integração à rede assistencial. A técnico-assistencial envolve os elementos do trabalho multidisciplinar, acolhimento, vínculo, processo de cuidar com enfoque familiar (Arantes, Shimizu \& MerchánHamann, 2016). Nessa circunstância, é relevante avaliar a ESF utilizando os componentes da estrutura (organização dos serviços), do processo (serviços produzidos) e de resultados (efetividade do serviço), com a finalidade de refletir possíveis intervenções na gestão e na prática assistencial.

Este estudo visou correlacionar os indicadores de ABS nos seus componentes de estrutura (cobertura populacional) com os de processo (consulta médica, visita domiciliar de médicos e enfermeiros, encaminhamento ao especialista, solicitação de exames de patologia clínica) e com os de resultado (taxa de internação por causas sensíveis à atenção básica, proporção de nascidos vivos de mães com sete ou mais consultas de pré-natal e coeficiente de mortalidade infantil) nos municípios do Estado de Mato Grosso, de 2008 a 2015, com a finalidade de promover a reflexão sobre a influência da ESF nos indicadores da ABS.

\section{Materiais e Métodos}

Trata-se de uma pesquisa avaliativa, retrospectiva, quantitativa, com a utilização de dados secundários obtidos pelos sistemas de informação relacionados à ESF. O período escolhido deve-se ao fato de que, em 2008, todos os municípios tinham aderido à ESF como organização e fortalecimento da Atenção Básica, mas somente em 2015 todos os dados do Sistema de Atenção Básica estavam completos.

A população deste estudo foi formada pelos 141 municípios de Mato Grosso. A amostra foi composta utilizando como critérios de inclusão: possuir equipes credenciadas e implantadas pelo MS no período compreendido entre janeiro e dezembro dos anos de 2008 a 2015 e estar com o SIAB cadastrado e implantado com as informações coletadas. Foram considerados excluídos da amostra os municípios com ausência de algum dado nos sistemas de informações e as unidades de saúde da família indígena.

Considerando-se as normativas do MS e o Pacto pela Vida, foram selecionados os seguintes indicadores de atenção básica disponíveis em sistemas de informação do MS: cobertura populacional, consultas médicas, visitas domiciliares de médicos e enfermeiros, encaminhamento ao especialista, solicitação de exames de patologia clínica, taxa de internação por causas sensíveis à atenção básica, número de nascidos vivos de mães com sete ou mais consultas de pré-natal e mortalidade infantil no estado de Mato Grosso. (Pellizzon, 2004) Os indicadores foram adaptados ao trabalho de Donabedian (1991) quanto aos componentes de Estrutura, Processo e Resultado, que estejam disponíveis em sistemas de informação do Ministério da Saúde.

O Quadro 1 apresenta os componentes de estrutura, de processo e de resultado com os indicadores de ABS, segundo o Pacto pela Vida adaptado do trabalho desse mesmo autor: 
Quadro 1 - Componentes e Indicadores da ESF.

\begin{tabular}{|c|c|}
\hline COMPONENTES & INDICADORES UTILIZADOS \\
\hline $\begin{array}{l}\text { Estrutura - são os recursos (materiais, humanos, financeiros e } \\
\text { técnicos) previamente disponíveis para a execução das atividades } \\
\text { de uma intervenção (projeto, programa ou serviço). }\end{array}$ & $\checkmark \quad$ Cobertura populacional potencial \\
\hline $\begin{array}{l}\text { Processo - é o conjunto de atividades durante as quais e pelas } \\
\text { quais os recursos são mobilizados e empregados pelos atores para } \\
\text { produzir bens e serviços requeridos para atender às finalidades do } \\
\text { sistema organizado da ação. }\end{array}$ & $\begin{array}{ll}\checkmark & \text { Média de Consulta médica por ano } \\
\checkmark & \text { Visita domiciliar por família por ano } \\
\checkmark & \text { Encaminhamento ao especialista por ano } \\
\checkmark & \text { Solicitação de exames de patologia clínica } \\
\text { por ano } & \end{array}$ \\
\hline $\begin{array}{l}\text { Resultado - são os efeitos intermediários da intervenção, } \\
\text { observados, em geral, na população-alvo. }\end{array}$ & $\begin{array}{lc} & \text { Taxa de internação por condições sensíveis } \\
\text { à atenção básica } \\
\checkmark \quad \text { Proporção de nascidos vivos de mães com } \\
\text { sete ou mais consultas de pré-natal } \\
\checkmark \quad \text { Coeficiente de Mortalidade Infantil }\end{array}$ \\
\hline
\end{tabular}

Fonte: Autores.

Segundo Donabedian (1991), o componente de estrutura mede a quantidade de todos os tipos de recursos que são aportados ao programa para viabilizar sua implementação. Neste estudo, foi selecionada a cobertura populacional potencial que se refere à proporção da população assistida pela equipe de saúde da família, tendo como referência 3.450 pessoas por equipe em determinado local e período. Foram analisadas as atividades de consulta médica, visita domiciliar, solicitação de exames de patologia clínica e encaminhamento ao especialista, sendo tais atividades diferenciadas dos demais modelos de atenção à saúde.

A consulta médica é considerada uma das principais atividades no trabalho cotidiano da equipe de saúde da família. O cálculo foi feito considerando o número de consultas médicas realizadas no ano e o denominador o número de habitantes no município, no mesmo ano. A visita domiciliar é uma tecnologia de interação no cuidado à saúde, sendo um instrumento de acompanhamento das famílias, fundamental para equipes como meio de conhecimento da realidade da população, estabelecimento de vínculos com a mesma e compreensão de aspectos importantes da dinâmica das relações familiares. O cálculo foi feito considerando a somatória do total de visitas médicas e de enfermeiros e o denominador o número de famílias multiplicado pelos meses no ano.

Buscando garantir a integralidade na assistência prestada, a ESF necessita de uma integração com as redes secundárias e terciárias para os encaminhamentos aos especialistas para diagnóstico, tratamento, internação e reabilitação. O cálculo foi feito considerando o número de encaminhamentos ao especialista no ano e o denominador o número de consultas médicas no ano.

Com base na integralidade na assistência prestada, faz parte das atividades desenvolvidas pelos médicos e enfermeiros a solicitação de exames de patologia clínica, entendida como exame complementar que tem por finalidade dar consistências às informações realizadas durante a consulta médica e de enfermagem através da anamnese e do exame físico. $\mathrm{O}$ cálculo adotado foi de acordo com o estabelecido pelo MS para avaliação de atendimento ambulatorial, o numerador foi considerado o número de solicitação de exames de patologia clínica no ano e o denominador o número de consulta médica no ano.

Na década de 1980, nos EUA, foi criado o indicador Ambulatory Care Sensitive Conditions - CSAP (Condições Sensíveis à Atenção Primária), para avaliar o impacto da falta de acesso a serviços na atenção primária. No Brasil, foi publicado através da Portaria MS/SAA ñ 221, de 17 de abril de 2008 (Brasil, 2008), uma Lista Brasileira de Internações por condições Sensíveis à AB. Em 2013, o MS atualizou e lançou o Caderno de Diretrizes, Objetivos, Metas e Indicadores para 2013-2015, no qual foram definidas as principais patologias: doenças evitáveis por imunização, gastroenterite de origem infecciosa presumível, anemia, deficiências nutricionais, infecções de nariz, ouvido e garganta, pneumonias bacterianas, asma, bronquites, hipertensão, angina, insuficiência cardíaca, diabetes mellitus, ,̧pilepsias, infecção no rim e no trato urinário, infecção da pele e 
tecido subcutâneo, doença inflamatória nos órgãos pélvicos femininos e síndrome da rubéola congênita. Para a composição dessa taxa de internação, foi feita a razão entre a soma das internações dessas doenças e o total de residentes no município no mesmo ano, multiplicado por cem, achando assim uma taxa de internações por condições sensíveis à atenção primária para um conjunto de mil habitantes/ano.

A proporção de nascidos vivos de mães com sete ou mais consultas de pré-natal contribui para evidenciar a qualidade da assistência pré-natal, o acesso ao serviço e identificando situações de desigualdades. Calculou-se, com base nos dados fornecidos pelo Sistema de Informação de Nascidos Vivos (SISNASC), a relação entre o número de gestantes que realizaram mais de sete consultas de pré-natal e o total de gestantes cadastradas no mesmo ano. De acordo com Starfierld (2009), o coeficiente de mortalidade infantil tem a capacidade de refletir o nível de saúde e desenvolvimento social; portanto, a sua indicação para avaliação em saúde tem sido altamente reconhecida. Para o cálculo do coeficiente de mortalidade infantil foi considerado o número de óbitos de residentes com menos de um ano de idade, dividido pelo número de nascidos vivos de mães residentes por 1.000 .

Os dados foram obtidos no Sistema de Informação da Atenção Básica (SIAB), Sistema de Informação sobre Nascidos Vivos (SINASC), Sistema de Informações Hospitalares do SUS (SIH-SUS), Sistema de Informação sobre Mortalidade (SIM), Cadastro Nacional de Estabelecimentos de Saúde (CNES) disponibilizados no site do Departamento de Informática do SUS (DATASUS) e Instituto Brasileiro de Geografia e Estatística (IBGE). A coleta de dados foi realizada entre março e abril de 2017 pelas autoras.

Os dados foram exportados e organizados em planilhas do Excel 2010 for Windows para o adequado armazenamento das informações. As análises estatísticas foram realizadas com o programa estatístico Statistical Package for the Social Sciences (SPSS) for Windows, versão 20.0. Foi realizado o teste de normalidade Shapirro Wilker que demonstrou que não há distribuição normal. Posteriormente, os dados foram analisados pelo Coeficiente de correlação de Spearman ( $\mathrm{r}_{\text {ho }}$ ) (Vieira, 2010) com o intuito de quantificar a correlação entre indicador de estrutura e os indicadores de processo e os indicadores de resultados. A análise estatística de todas as informações coletadas nesta pesquisa foi inicialmente feita de forma descritiva através da média, mediana, valores mínimo e máximo, desvio padrão e frequência absoluta e percentual. Foi feita a descrição dos dados, analisando todos os municípios e indicadores.

O projeto foi analisado e aprovado pelo Comitê de Ética em Pesquisa da UNIFESP/EPM sob o protocolo 691.439.

\section{Resultados}

De um total de 141 municípios em Mato Grosso, 106 (75,2\%) apresentaram informações completas, permitindo a análise dos indicadores selecionados. Ao analisar o porte do município, observa-se que 98 (92,5\%) são de pequeno porte (até 50.000 habitantes), 05 (4,7\%) de médio porte (até 500.000 habitantes) e 03 (2,8\%) de grande porte (mais de 500.001 habitantes).

Quanto ao comportamento dos indicadores da atenção básica, segundo o componente de estrutura, a média da cobertura populacional da ESF, em 2008 , era de $83,33 \%$, aumentando em $87,69 \%$ para 2015 , ou seja, um aumento percentual de 5,23\% entre o período de 2008 e 2015.

Segundo o componente de processo, a média de consulta médica era de 1,19 consultas médicas/residente/ano em 2008, aumentando para 1,75 em 2012 e reduzindo para 1,55 em 2015. Em relação à visita domiciliar, alguns municípios apresentaram o valor mínimo menor que uma visita domiciliar/família/ano. A média da visita domiciliar variou de 23 visitas domiciliares/família/ano em 2008, caindo para 21,55 em 2012 e declinando para 21,36 em 2015, com variância de 47,96 no período. No que concerne ao encaminhamento ao especialista, a média variou de 0,19 encaminhamento ao especialista/consulta médica/ano em 2008, caindo para 0,09 em 2011, 2012 e 2013 e reduzindo para 0,07 em 2015. Em relação à solicitação de 
exames de patologia clínica, a média era de 1,73 solicitações de exames de patologia clínica/consulta médica/ano em 2008, caiu para 0,51 em 2009, aumentando para 0,94 em 2013 e teve um leve declínio para 0,87 em 2015.

Segundo o componente de resultado, no que se refere à Taxa de Internação por causas sensíveis à atenção básica (TICSAB), a média apresentou redução no período estudado, sendo 35,61\% em 2008, 32,91\% em 2012 e reduzindo para 28,81\% em 2015. No tocante à Proporção de Nascidos vivos de mães com sete ou mais consultas de pré-natal, o valor máximo desse indicador se manteve alto no período, em 2008 era 95,37\%, aumentando em 2012 para 100\% e sofrendo uma leve queda para 96,90\% em 2015. Com relação ao Coeficiente de Mortalidade Infantil, a média reduziu gradativamente, em 2008 era de 20,30/mil nascidos vivos, caindo para 14,31 em 2012 e chegando a 13,91 em 2015.

Para verificar a correlação dos indicadores de estrutura-processo e estrutura-resultado, se é positiva ou negativa e o comportamento durante os anos de 2008 a 2015, foi realizado o Coeficiente de correlação de Spearman ( $\mathrm{r}_{\text {ho }}$ ) entre o componente de estrutura e o componente de processo e, posteriormente, entre componente de estrutura e componente de resultado. Esse coeficiente expressa um valor de $r_{\text {ho }}$ entre $-1,00$ e $+1,00$, indicando uma associação (negativa ou positiva). No caso de uma correlação positiva, a variável $x$ se dá na mesma proporção que a variável $y$; já no caso de uma correlação negativa, à medida que a variável $x$ cresce a variável $y$ decresce (Vieira, 2010).

A Tabela 1 apresenta os seguintes resultados: correlação positiva entre cobertura populacional e média de consultas médicas, ou seja, à medida que aumenta um há aumento também da outra. O mesmo ocorreu com a cobertura populacional e visita domiciliar por família. Entre a cobertura populacional e encaminhamento ao especialista houve uma correlação negativa, indicando que à medida que aumenta uma há redução na outra. O mesmo ocorreu na correlação entre cobertura populacional e solicitação de exames de patologia clínica.

Tabela 1 - Matriz de correlação do componente de estrutura versus componente de processo da Estratégia Saúde da Família nos municípios de Mato Grosso, 2018.

\begin{tabular}{|c|c|c|c|}
\hline \multirow[b]{2}{*}{ Estrutura } & \multicolumn{3}{|c|}{ Processo } \\
\hline & $\begin{array}{c}\text { Taxa de Interuacio por } \\
\text { Causas Sensiveis a } \\
\text { Atencio Basica }\end{array}$ & $\begin{array}{c}\text { Percentual de Nascidos Vivos } \\
\text { de màes com sete ou mais } \\
\text { consultas de Pré-Natal }\end{array}$ & $\begin{array}{c}\text { Coeficiente de } \\
\text { Mortalidade Infanta }\end{array}$ \\
\hline Coberturs Populacional & $\begin{array}{c}0,0252 \\
\text { phos: } 0.4631\end{array}$ & $\begin{array}{c}0.0114 \\
\text { prap: } 0.740\end{array}$ & $\begin{array}{c}-0,0317 \\
\text { pap: } 0,356\end{array}$ \\
\hline
\end{tabular}

Fonte: Dados da pesquisa.

Conforme a Tabela 2, realizou-se também a correlação entre o componente de estrutura e de resultado através do Coeficiente de correlação de Spearman ( $\mathrm{r}_{\mathrm{ho}}$ ) para identificar se existe correlação e caso afirmativo, se é positiva ou negativa. Existe correlação positiva entre cobertura populacional e taxa de internação por Causas Sensíveis à Atenção Básica por ano, assim como, entre cobertura populacional e percentual de nascidos vivos de mães com sete ou mais consultas de pré-natal por ano. Ao contrário, existe uma correlação negativa entre cobertura populacional e Coeficiente de Mortalidade Infantil por ano. 
Tabela 2 - Matriz de correlação do componente de estrutura versus componente de resultado da Estratégia Saúde da Família nos municípios de Mato Grosso, 2018.

\begin{tabular}{|c|c|c|c|c|}
\hline \multirow[b]{2}{*}{ Estrufura } & \multicolumn{4}{|c|}{ Processo } \\
\hline & $\begin{array}{l}\text { Consulta } \\
\text { Médica }\end{array}$ & $\begin{array}{c}\text { Visita } \\
\text { Domiciliar }\end{array}$ & $\begin{array}{c}\text { Encaminhamento ao } \\
\text { Especialista }\end{array}$ & Solicitasào exames \\
\hline Cobertura Populacional & $\begin{array}{c}0.4847 \\
\text { pdta: } 0,000\end{array}$ & $\begin{array}{c}0.4202 \\
\mathrm{paw}: 0,000\end{array}$ & $\begin{array}{c}-0,0984 \\
\text { pitu: } 0,004\end{array}$ & $\begin{array}{c}0,0527 \\
\text { Pits } 0,1248\end{array}$ \\
\hline
\end{tabular}

Fonte: Autores.

\section{Discussão}

Buscou-se verificar a influência da ESF nos indicadores da ABS em Mato Grosso ao conhecer a situação daquela população e ao discutir seus resultados no intuito de contribuir para a consolidação da ESF, apontando se houve ou não mudanças nas práticas assistenciais e nas condições de saúde dos indivíduos.

Ao refletir sobre os resultados do comportamento dos indicadores de atenção básica na ESF, com relação ao componente de estrutura, verificou-se que sempre se manteve alta a proporção da cobertura populacional pela equipe de saúde da família, sendo acima de $80 \%$ ao longo dos oito anos estudados, corroborando, com os resultados obtidos nos estudos de Malta et al. (2016) e Silva (2011) que apontaram aumento da cobertura populacional acima de $80 \%$ nos estados de Tocantins, Paraíba e Piauí, em áreas rurais e em municípios de pequeno porte.

Com o aumento da cobertura populacional da ESF é esperada a ampliação do acesso da população aos serviços de saúde, pois segundo o MS essa é a porta de entrada aos serviços de saúde incorporando melhoria nos cuidados recebidos, refletindo nos indicadores de saúde: redução na mortalidade infantil, detecção precoce de cânceres, menor mortalidade devido às causas previsíveis e aumento da expectativa de vida (Binda, Bianco \& Sousa, 2013; Malta et al., 2016).

Segundo essa premissa e considerando o componente de processo como atividades que envolvem a equipe multiprofissional, a pesquisa buscou explorar os indicadores com a finalidade de encontrar rupturas com o modelo assistencial centrado na doença. Os indicadores consultas médicas, visita domiciliar, encaminhamentos ao especialista considerando a organização do sistema de referência e a solicitação de exames de patologia clínica melhoram com a implantação e a evolução da ESF nos municípios (Brasil, 2010).

Assim, percebem-se algumas divergências no comportamento dos indicadores de processo. Houve um aumento (30,25\%) na média de consultas médicas nos oito anos estudados, o que sugere melhora no acesso ao serviço da atenção básica. Em contrapartida, a média de visitas domiciliares por família por ano, realizadas pelo médico e enfermeiro, diminuiu ao longo do tempo (-7,13\%). Por outro lado, a média de encaminhamentos ao especialista, teve uma redução significativa entre os anos de 2008 e 2015 (-63,16\%), o que pode nos leva a pensar que a ESF conseguiu resolver os problemas de saúde na atenção básica. O mesmo fenômeno ocorreu com a solicitação de exames de patologia clínica, com redução (-49,71\%). A consulta médica na ESF busca aumentar e fortalecer o vínculo "paciente e profissional”, é focada em compreender as necessidades do indivíduo, e não somente a doença, com participação e negociação com a pessoa que está sendo cuidada, realizando uma abordagem compreensiva no manejo da enfermidade e de sua família (Ramos, 2008).

Os resultados obtidos na pesquisa mostram que, com o aumento da cobertura populacional a ESF proporcionou um aumento da demanda da população por atendimento médico. Contudo, essa demanda inviabiliza que a equipe multiprofissional exerça outras atividades previstas que provocam mudanças nas práticas assistenciais da comunidade estudada e consigam, assim, 
dar respostas positivas aos problemas de saúde.

Quanto à solicitação de exames de patologia clínica por consulta médica por ano para auxiliar no diagnóstico médico e acompanhamento do tratamento, no Brasil, são raros os estudos que abordam diretamente a solicitação de exames médicos, sendo fragmentados e não condizentes com a realidade do sistema de saúde como um todo (Brasil, 2002). A reflexão desse componente de processo nos levou a reconhecer que, para que haja mudança no modelo de saúde hegemônico, é preciso que ocorram mudanças de comportamento de todos os envolvidos, passando pelos gestores, pelos profissionais de saúde e chegando aos usuários. Para que isso aconteça, é necessário modificar a formação dos profissionais de saúde que futuramente estarão trabalhando na ESF.

Quanto ao componente de resultado, foram escolhidos três indicadores que estão relacionados ao acesso, à morbidade e à mortalidade, que são potencialmente influenciadas pela presença da ABS. Os resultados apresentados descrevem um comportamento positivo destes indicadores, apontando declínio na taxa de internação por causas sensíveis à atenção primária, aumento da proporção de nascidos vivos de mães com sete ou mais consultas no pré-natal e redução do coeficiente de mortalidade infantil. Comparando o valor máximo da TICSAB em 2008 (79,74\%) e o valor máximo em 2015 (73,33\%), houve redução de $8,04 \%$ das internações. A média se manteve acima de $30 \%$ ao longo do período estudado.

Este estudo apresenta limitações por considerar apenas dados secundários oriundos dos sistemas oficiais de informação do MS e, assim, sujeitos aos possíveis vieses relacionados à qualidade dos dados disponíveis, embora seja observada melhoria da qualidade das informações e a grande disponibilidade dessas que podem também contribuir para o aumento dos valores em determinados momentos. Além disso, ao analisar esse indicador, é preciso considerar as condições de vida da população estudada como perfil sócio demográfico e econômico das populações, as situações epidemiológicas específicas, a própria organização da rede de serviços existentes e as atividades das equipes de ESF em especial.

Estudo semelhante com a utilização de dados secundários, realizado por Moura et al. (2010) com dados do Sistema de Informação Hospitalar, no período de 1999 a 2006, analisando as tendências das principais causas de internações hospitalares sensíveis à atenção primária, demonstrou a redução das taxas de internação por gastroenterite $(-12,0 \%)$ e asma $(-31,8 \%)$ e aumento de $142,5 \%$ nas taxas de internações por pneumonias bacterianas.

Outro indicador utilizado para análise da qualidade da assistência à saúde materno-infantil no âmbito da atenção básica se refere à assistência pré-natal, seguindo os critérios do Programa de Humanização do Parto e Nascimento, implantado pelo MS no ano de 2000, que tem como proposta aumentar e melhorar o acesso e acolhimento precoce das gestantes, garantindo o número mínimo de seis consultas de pré-natal, e uma série de outras ações assegurando diminuir a morbimortalidade materno infantil (Oyerinde, 2013; Lansky et al., 2014; Tomasi et al., 2017). Neste estudo foi avaliada a proporção de nascidos vivos de mães com sete ou mais consultas de pré-natal. Percebeu-se que esse indicador apresentou valores acima de $90 \%$ durante o período estudado, com 1,60\% de aumento entre os anos de 2008 e 2015. Esses dados são satisfatórios, podendo inferir que as gestantes estão tendo acesso e sendo acompanhadas pelos profissionais da saúde da família.

Verifica-se o mesmo fenômeno de positividade ao analisar o Coeficiente de Mortalidade Infantil no período estudado que apresentou declínio de 31,51\%. No Brasil, no ano de 2011, a taxa de mortalidade infantil foi 15,3 por mil nascidos vivos, atingindo a Meta 4 dos Objetivos de Desenvolvimento do Milênio, compromisso dos governos integrantes das Nações Unidas de melhorar a saúde infantil e reduzir em 2/3 a mortalidade infantil entre 1990 e 2015 (Maranhão et al., 2012; Murray et al., 2007).

Ao correlacionar os componentes de estrutura e de processo, a fim de analisar o desempenho da ESF na atenção básica, observou-se que é possível afirmar que a Cobertura Populacional influencia nestes indicadores. Entre os componentes de estrutura e processo, observou-se que há correlação entre os indicadores e constata-se que essa é positiva entre cobertura populacional, consulta médica e visita domiciliar. Esses dado̧ se alinham com a proposta da ESF, tendo como atribuições 
comuns a todos os profissionais da equipe realizar ações de saúde à população prioritariamente nas unidades de saúde e, quando necessário, no domicílio, garantindo o cuidado integral, contínuo e organizado (Brasil, 2012). Pode-se dizer que, para que ocorra a implementação da ESF é preciso que o município se organize nas ações e na oferta de serviços, garantindo a presença de todos os profissionais necessários para a atenção básica, para que consiga garantir o acesso aos serviços e à oferta de qualidade.

Santos (2013), em seu estudo, identificou que a implementação da ESF não está relacionada diretamente com o porte habitacional dos municípios. Seus efeitos positivos estão condicionados ao aumento do acesso aos serviços de saúde desde o nível mais elementar até os mais complexos do sistema de saúde. Um estudo realizado com municípios de pequeno porte de uma microrregião da Bahia no ano de 2011, apontou pontos positivos nos aspectos político-institucionais, como projetos de governo garantindo a universalização da ESF, iniciativas de desprecarização do trabalho em saúde, reconhecimento da necessidade de capacitação das equipes de saúde da família e investimentos em saúde. Todavia, apontaram dificuldades no planejamento das ações gerenciais e assistenciais, formulação dos instrumentos de gestão e nas práticas de avaliação e controle social (Silva, 2011).

$\mathrm{Na}$ análise da correlação entre os componentes de estrutura e resultado, apontou-se que há correlação entre os indicadores, sendo positiva entre cobertura populacional e taxa de internação por causas sensíveis à atenção básica e proporção de nascidos vivos de mães com sete ou mais consultas de pré-natal. Verificou-se também correlação negativa entre cobertura populacional e coeficiente de mortalidade infantil.

A utilização desses indicadores representa um importante instrumento para avaliar a implementação da ESF, apresentando análise situacional, epidemiológica da área pesquisada, colaborando para a definição de intervenções prioritárias locais de saúde.

\section{Conclusão}

A pesquisa permitiu avaliar que, com a evolução da ESF ocorreram alterações em alguns indicadores de atenção básica à saúde quando correlacionados segundo seus componentes de estrutura, de processo e de resultado, no período de 2008 a 2015. Os resultados demonstraram melhora na acessibilidade ao serviço, na efetividade positiva das atividades desenvolvidas pelas equipes de saúde da família, porém não é possível afirmar que houve mudança no modelo assistencial.

Já, quanto ao efeito do programa, demonstrou um melhor comportamento, sugestivo de mudanças nas condições de saúde dos indivíduos. Ao correlacionar os componentes de estrutura, de processo e de estrutura e resultado, observou-se que há uma associação significativa para sugerir que a ESF no Estado de Mato Grosso proporcionou melhora nos indicadores da atenção básica no período estudado.

Entre os indicadores analisados na pesquisa, a correlação entre cobertura populacional e taxa de internação por causas sensíveis à atenção básica apresentou resultado divergente de outros estudos. Este pode estar associado a vícios nas informações coletadas, ou até mesmo ao comportamento dos profissionais em estar influenciando nas indicações de internação. Sugere-se outras pesquisas mais aprofundadas.

O tipo de análise sugerida no estudo e, também, para futuras pesquias científicas focadas nesse tema, contribui como ferramenta de gestão na análise dos indicadores processados pelas equipes de saúde da família, utilizando-se como feedback da gestão para os profissionais de saúde e estes discutindo entre si para planejamento de intervenções para melhoria da saúde de população delimitada.

\section{Agradecimentos}

Este estudo foi financiado pela CAPES. É produto parcial de pesquisa de Doutorado desenvolvida na Escola Paulista de Medicina, da Universidade Federal de São Paulo, e aprovada pelo Comitê de Ética em Pesquisa da Universidade. 


\section{Referências}

Arantes, L. J., Shimizu, H. E., \& Merchán-Hamann, E. (2016). Contribuições e desafios da ESF na Atenção Primária à Saúde no Brasil: revisão da literatura. Ciência \& Saúde Coletiva,21(5),1499-1510.

Binda, J., Bianco, M., \& Sousa, E. (2013). O trabalho dos agentes comunitários de saúde em evidência: uma análise com foco na atividade. Saúde e Sociedade, 22(2),389-402.

Brasil. (2002) Ministério da Saúde. Indicadores básicos de saúde no Brasil: Conceitos e aplicações. OPAS.

Brasil. (2008). Ministério da Saúde. Portaria GM n 221 de 17 de abril de 2008. Define Lista Brasileira de Internações por Condições Sensíveis à Atenção Primária. Diário Oficial da União: República Federativa do Brasil.

Brasil. (2010). Ministério da Saúde. Departamento de Atenção Básica. Memórias da saúde da família no Brasil.

Brasil. (2012). Ministério da Saúde, Secretaria de Atenção à Saúde. Política Nacional de Atenção Básica. Brasília: Ministério da Saúde.

Donabedian, A. (1991). La calidad de la atención médica: definición y métodos de evaluación. Copilco.

Lansky, S., Friche, A. A. de L., Silva, A. A. M. da, Campos, D., Bittencourt, S. D. de A., Carvalho, M. L. de et al. (2014). Pesquisa Nascer no Brasil: perfil da mortalidade neonatal e avaliação da assistência à gestante e ao recém-nascido. Cad. Saúde Pública, 30(1),192-207.

Malta, D., Santos, M., Stopa, S., Vieira, J., Melo, E., \& Reis A. (2016). A Cobertura da Estratégia de Saúde da Família (ESF) no Brasil, segundo a Pesquisa Nacional de Saúde, 2013. Ciência \& Saúde Coletiva;21(2):327-338.

Maranhão, A. G. K., Vasconcelos, A. M. N., Porto, D. L., França, E. et al. (2012). Mortalidade infantil no Brasil: tendências, componentes e causas de morte no período de 2000 a 2010. In: Departamento de Análise de Situação de Saúde, Secretaria de Vigilância em Saúde, Ministério da Saúde, organizador. Saúde Brasil 2011: uma análise da situação de saúde e a vigilância da saúde da mulher. v. 1. Brasília: Ministério da Saúde.

Motta, L. C. S., \& Siqueira-Batista, R. (2015) ESF: clínica e críticas. Rev Bras Edu Med., 39(2),196-207.

Moura, B. L. A., Cunha, R. C. da, Aquino, R., Medina, M. G., Mota, E. L. A., Macinko, J. et al. (2010). Principais causas de internação por condições sensíveis à atenção primária no Brasil: uma análise por faixa etária e região. Rev. Bras. Saúde Mater. Infant., 10(1), 83-91.

Murray, C. J., Laakso, T., Shibuya, K., Hill, K., \& Lopez, A. D. (2007). Can we achieve Millennium Development Goal 4? New analysis of country trends and forecasts of under-5 mortality to 2015. Lancet. 370:1040-54.

Oyerinde, K. (2013). Can antenatal care result in significant maternal mortality reduction in developing countries? J Community Med Health Educ, 3:116.

Pellizzon, R. D. F. (2004). Pesquisa na área da saúde: 1. Base de dados DeCS (Descritores em Ciências da Saúde). Acta Cirúrgica Brasileira, $19,153-163$.

Ramos, V. (2008). A Consulta em 7 Passos. Book.

Sala, A., \& Mendes, J. D. V. (2011). Perfil de indicadores da atenção primária à saúde no estado de São Paulo: retrospectiva de 10 anos. Saúde soc.,20(4),912926.

Santos, P. F. B. B. (2013). Avaliação do Programa Saúde da Família no Rio Grande do Norte. Tese.

Senna, M. H., \& Andrade, S. R. de. (2015). Indicators and Information in Local Health Planning: the perspective of the Family Health Strategy Nurses. Texto contexto - enferm., 24(4),950-958.

Silva, L. A. (2011). Avaliação do grau de implantação da ESF em municípios de pequeno porte. Dissertação

Soratto, J., Pires, D. E. de, Dornelles, S., Lorenzetti, J. et al (2015). Family health strategy: a technological innovation in health. Texto \& Contexto Enfermagem, 24(2),584-592.

Starfield, B. (2002). Atenção primária: equilíbrio entre necessidades de saúde, serviços e tecnologia. UNESCO.

Tomasi, E., Fernandes, P. A. A., Fischer, T., Siqueira, F. C. V., Silveira, D. S. da, Thumé, E. et al. (2017). Qualidade da atenção pré-natal na rede básica de saúde do Brasil: indicadores e desigualdades sociais. Cad. Saúde Pública, 33(3),e00195815

Vieira, S. (2010). Introdução à Bioestatística. Book. 